\title{
Transparent Metal-Organic Framework-Based Gel Electrolytes for Generalized Assembly of Quasi-Solid-State Electrochromic Devices
}

Zhiyuan Bai ${ }^{\sharp a}$, Ran Li ${ }^{\# a}$, Kerui Li ${ }^{* a, b}$, Chengyi Hou ${ }^{a}$, Qinghong Zhang ${ }^{c}$, Yaogang $\mathrm{Li}^{c}$, Hongzhi Wang*a

${ }^{a}$ State Key Laboratory for Modification of Chemical Fibers and Polymer Materials, College of Materials Science and Engineering, Donghua University, Shanghai 201620, People's Republic of China

b Department of Chemical and Biomolecular Engineering, National University of Singapore, 117585, Singapore

c Engineering Research Center of Advanced Glasses Manufacturing Technology, Ministry of Education, Donghua University, Shanghai 201620, People's Republic of China

\#These authors equally contributed to this work.

Corresponding Author

* Email: chelik@ nus.edu.sg (Kerui Li)

* Email: wanghz@dhu.edu.cn (Hongzhi Wang)

KEYWORDS: electrochromic devices, metal-organic frameworks, gel electrolytes, high transparency, large ion channels 


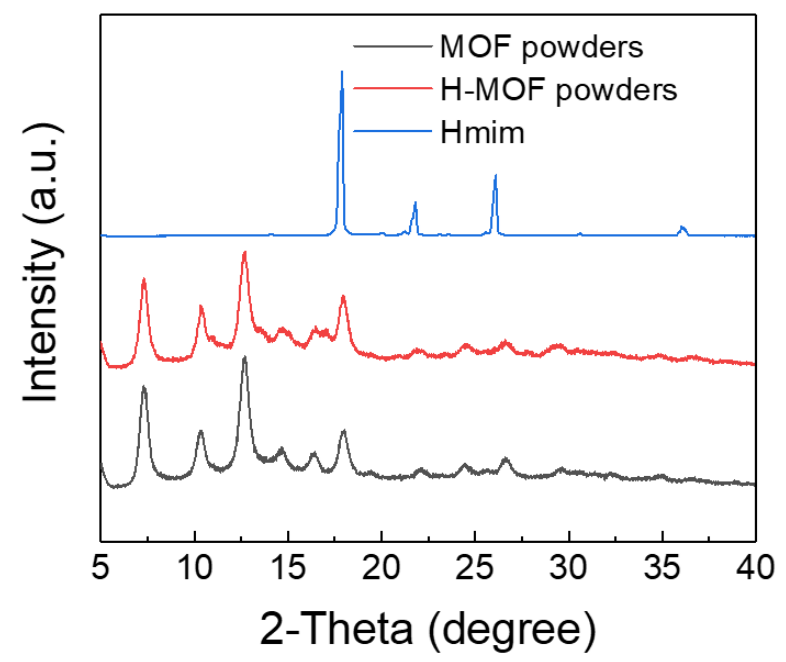

Figure S1. XRD patterns of MOF, H-MOF, and Hmim powders.

a

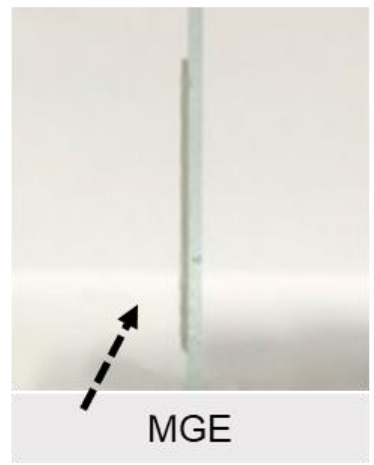

b

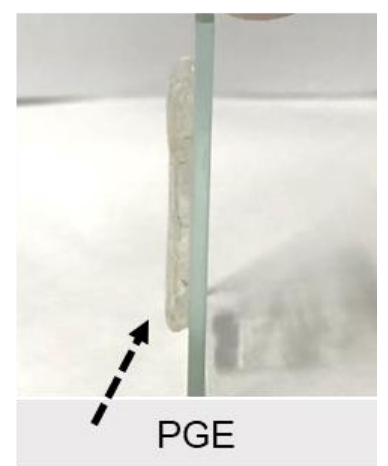

Figure S2. (a) Digital photograph of the transparent MOF-based gel electrolyte (MGE) coated on a vertical glass slide, showing typical gel state. (b) Digital photograph of the PMMA-based gel electrolyte (PGE). 


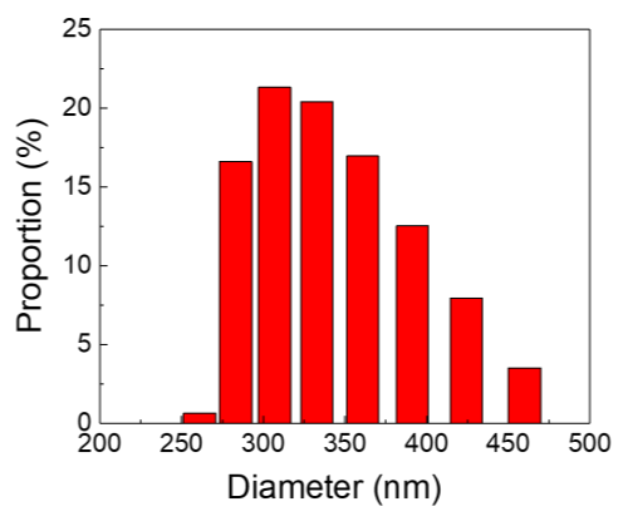

Figure S3. Size distribution of agglomerated MOF particles in PC solvent measured through a particle size analyzer.

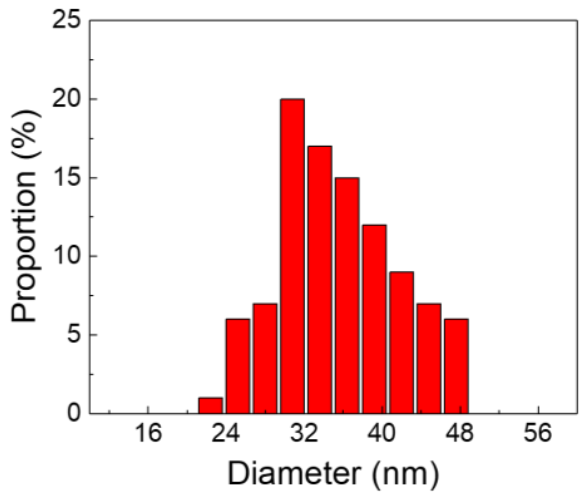

Figure S4. Size distribution of MOF nanocrystals calculated according to the SEM image. 

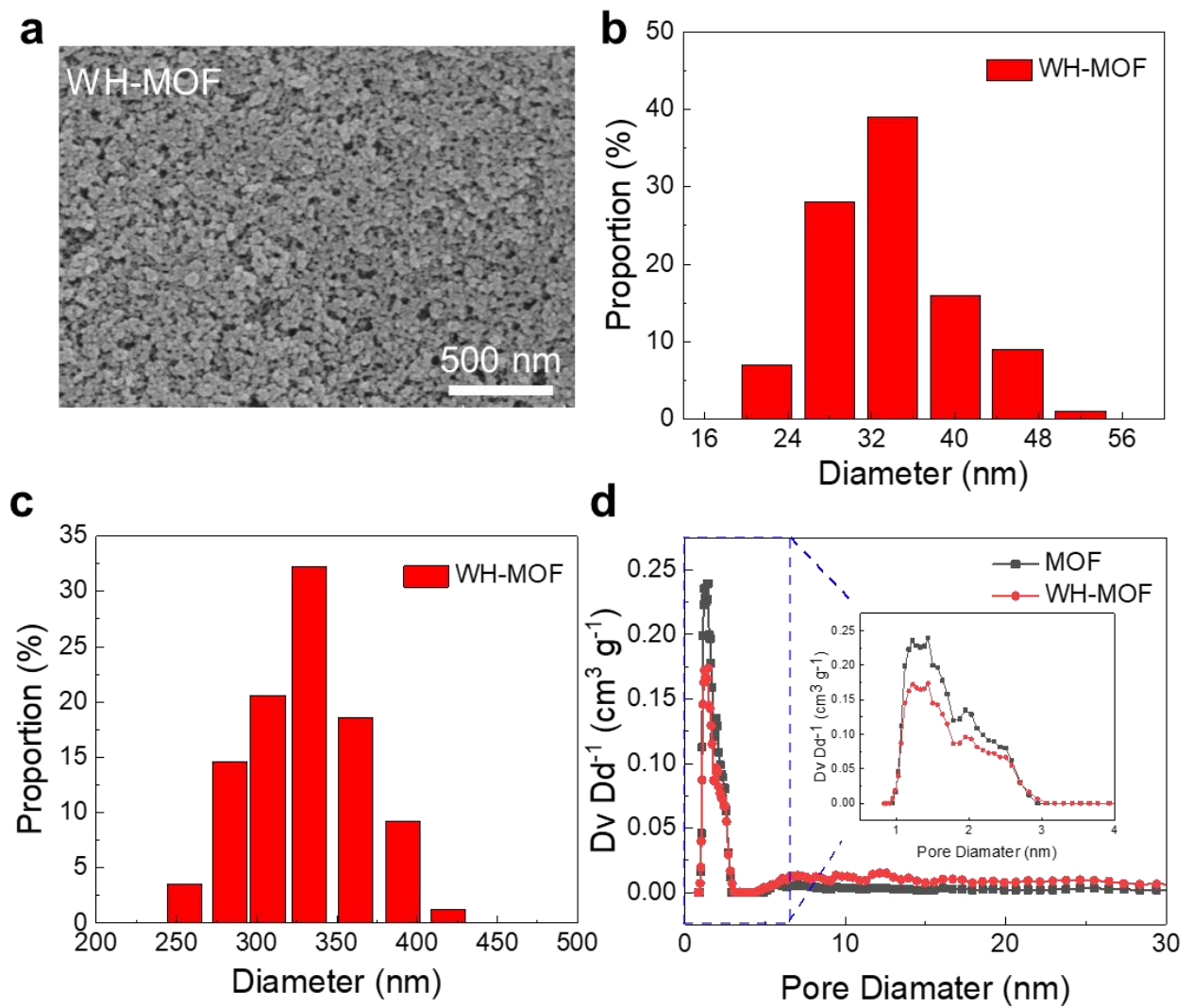

Figure S5. (a) FE-SEM image of WH-MOF. (b) Size distribution of WH-MOF nanocrystals estimated according to the SEM image. (c) Size distribution of WH-MOF particles in PC solvent measured using a particle size analyzer. (d) Pore size distribution of MOF and WH-MOF.

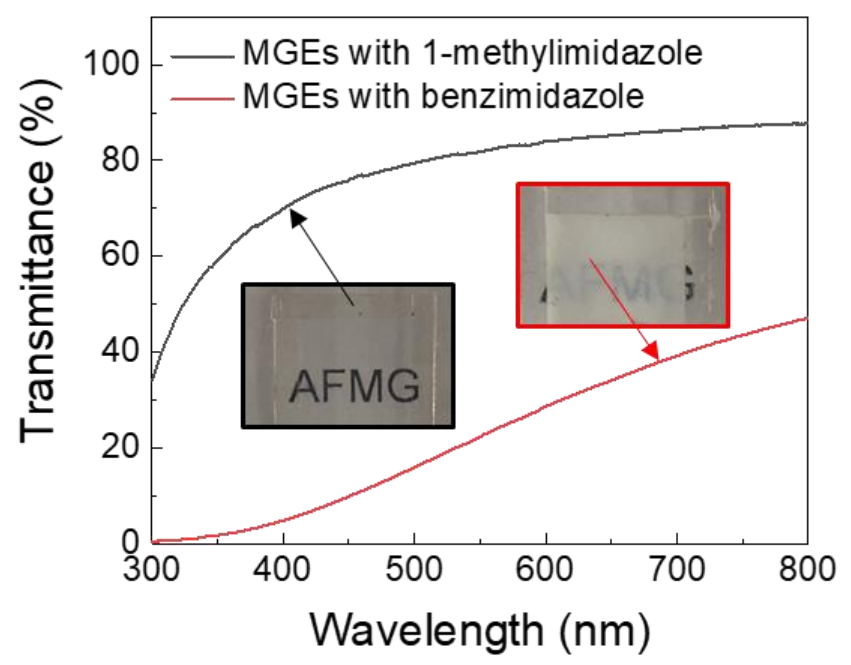

Figure S6. UV-vis transmittance spectra of MGEs with 1-methylimidazole and benzimidazole, respectively. Insets are the corresponding digital photographs. 


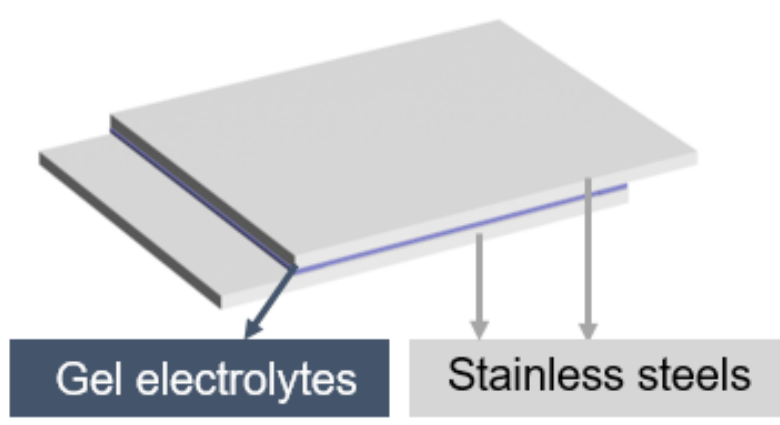

Figure S7. Schematic illustration of setup for electrochemical impedance spectroscopy (EIS) testing. Here, stainless steel plates were used to sandwich gel electrolytes.

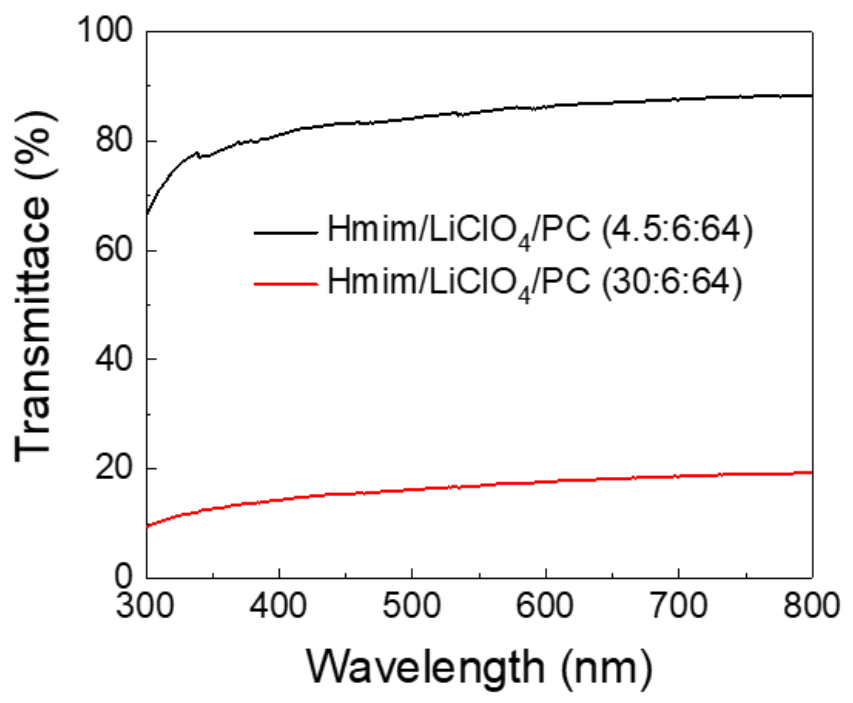

Figure S8. UV-vis transmittance spectra of $\mathrm{LiClO}_{4} / \mathrm{PC}$ with different amounts of Hmim. 


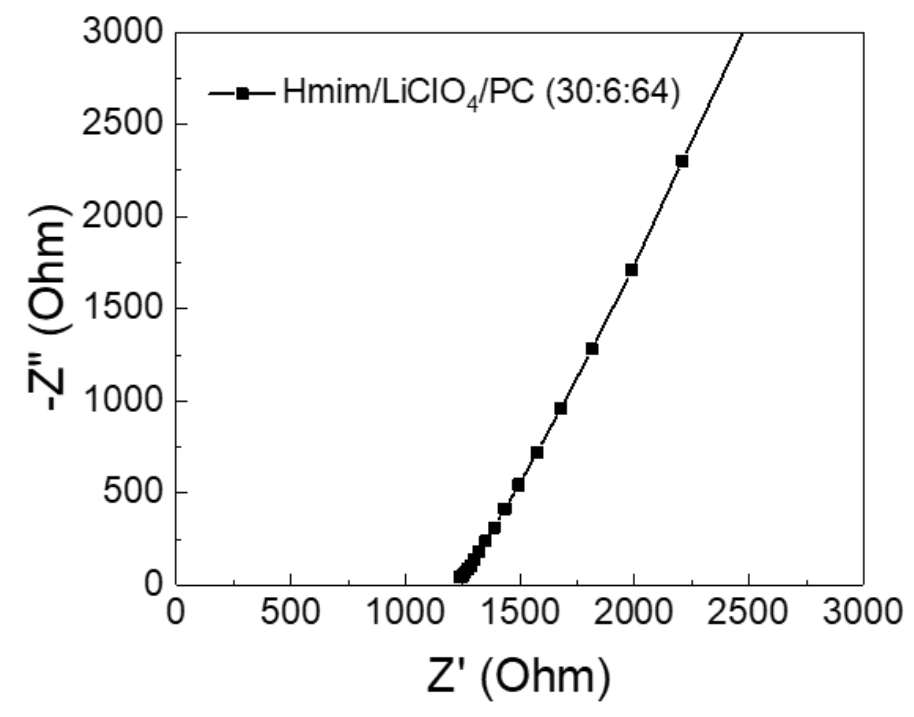

Figure S9. Electrochemical impedance spectroscopy (EIS) plots of $\mathrm{Hmim} / \mathrm{LiClO}_{4} / \mathrm{PC}$ (30:6:64).
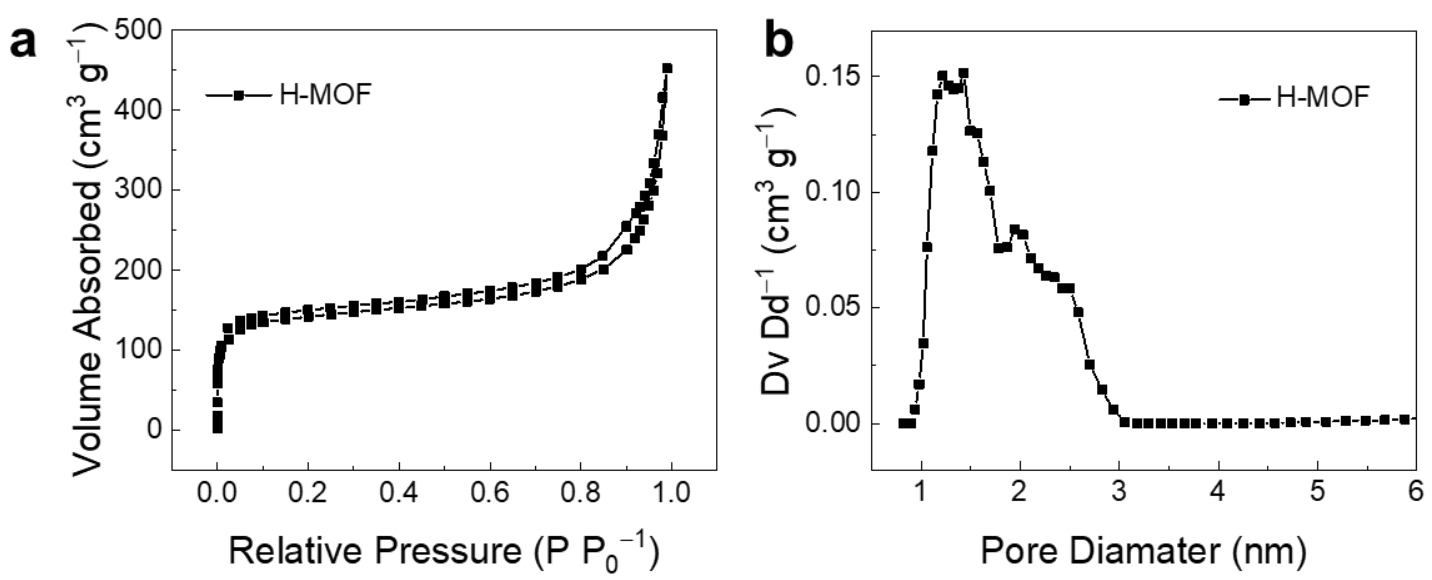

Figure S10. (a) $\mathrm{N}_{2}$ adsorption isotherm curves of H-MOF. (b) Pore size distribution of H-MOF. 


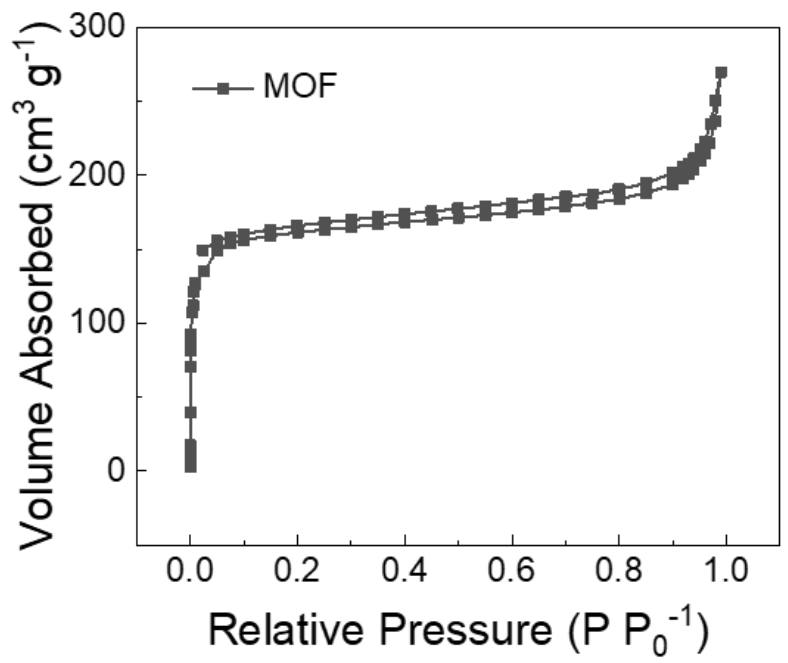

Figure S11. $\mathrm{N}_{2}$ adsorption isotherm curves of MOF.

The specific surface area of MOF was calculated to be $620.3 \mathrm{~cm}^{2} \mathrm{~g}^{-1}$. The pore volume was ca. $0.372 \mathrm{~cm}^{3} \mathrm{~g}^{-1}$. The pore size of pristine MOF was concentrated in ca. $14.3 \AA$.

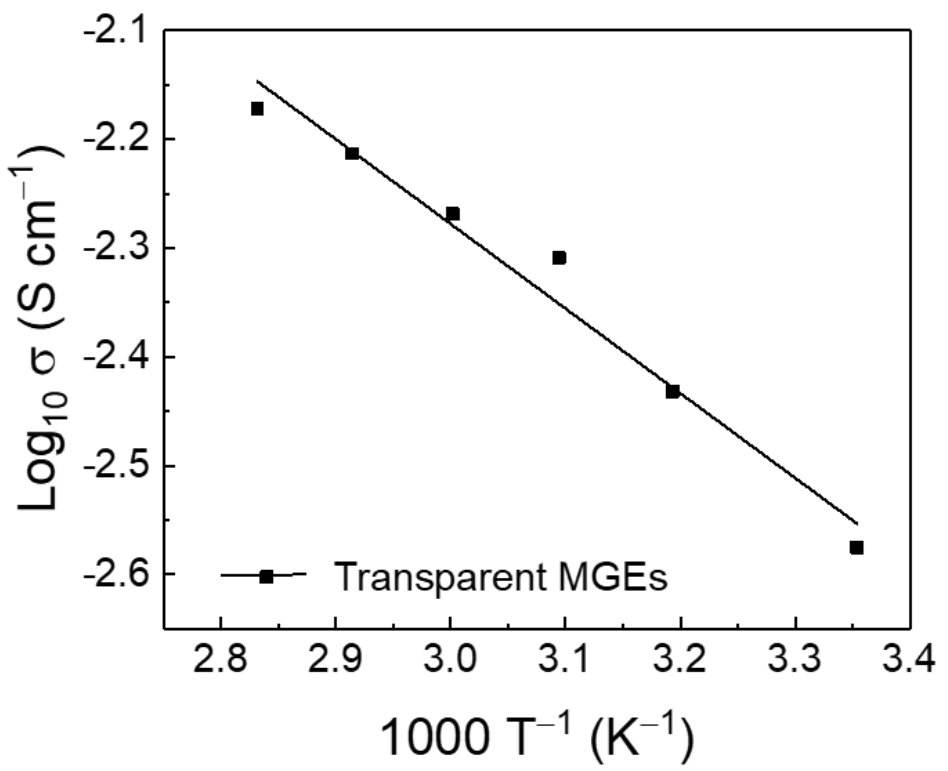

Figure S12. Arrhenius plot of the transparent MGEs. 

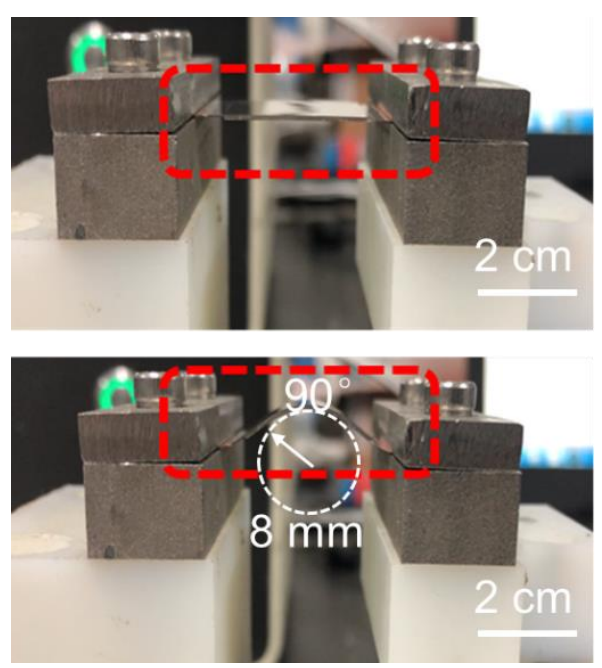

Figure S13. Digital photograph of the setup for the bending test of flexible devices under a bending angle of $90^{\circ}$ and curvature radius of $8 \mathrm{~mm}$.
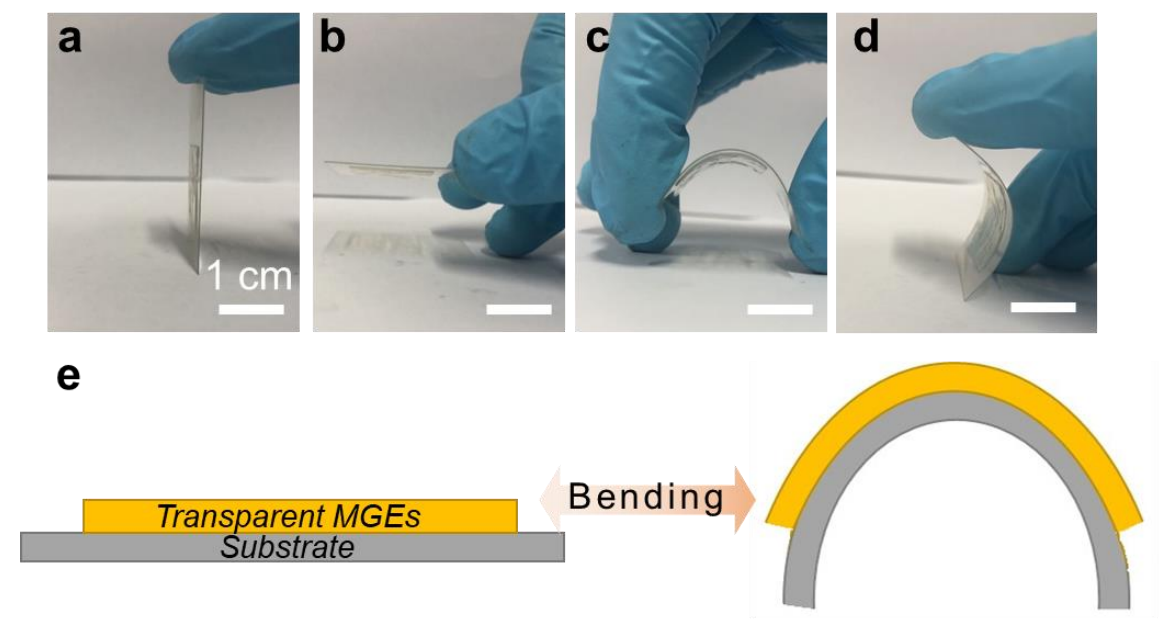

f
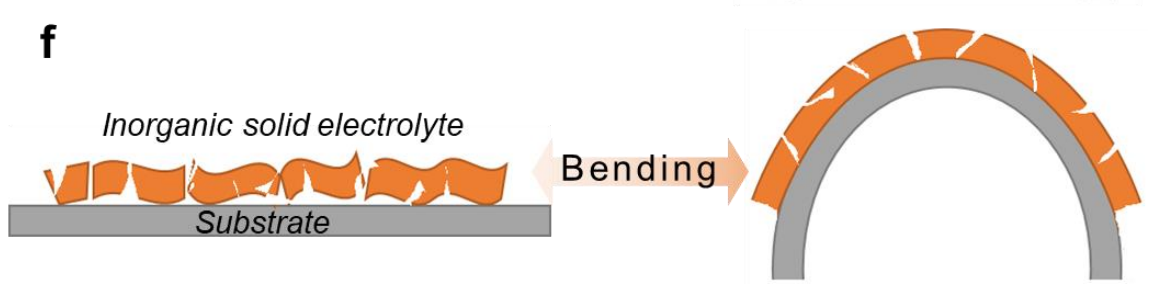

Figure S14. Digital photographs of the transparent MGE coated on the flexible ITOPET flim under different states: (a) vertical state, (b) inverted state, and (c-d) bending states. Schematic illustrations of (e) the transparent MGEs and (f) traditional solid inorganic electrolyte under bending state. 

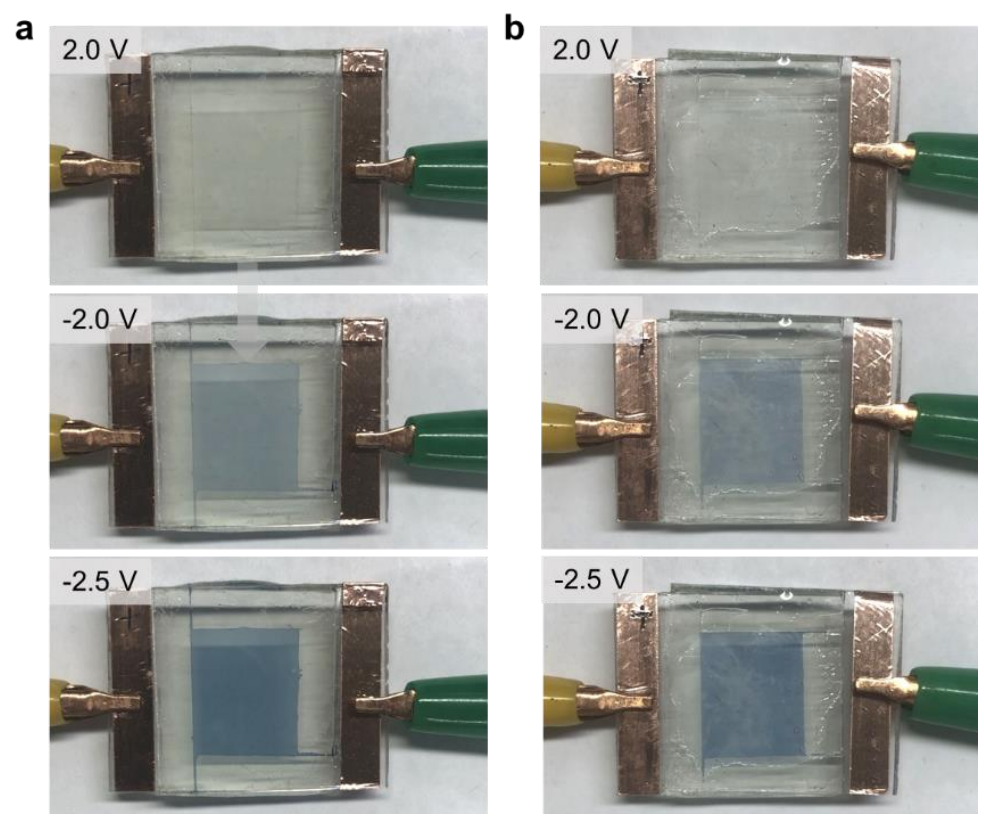

Figure S15. Digital photographs of $\mathrm{WO}_{3}$-based electrochromic (EC) devices assembled using (a) transparent MGEs and (b) PGEs under different voltages.

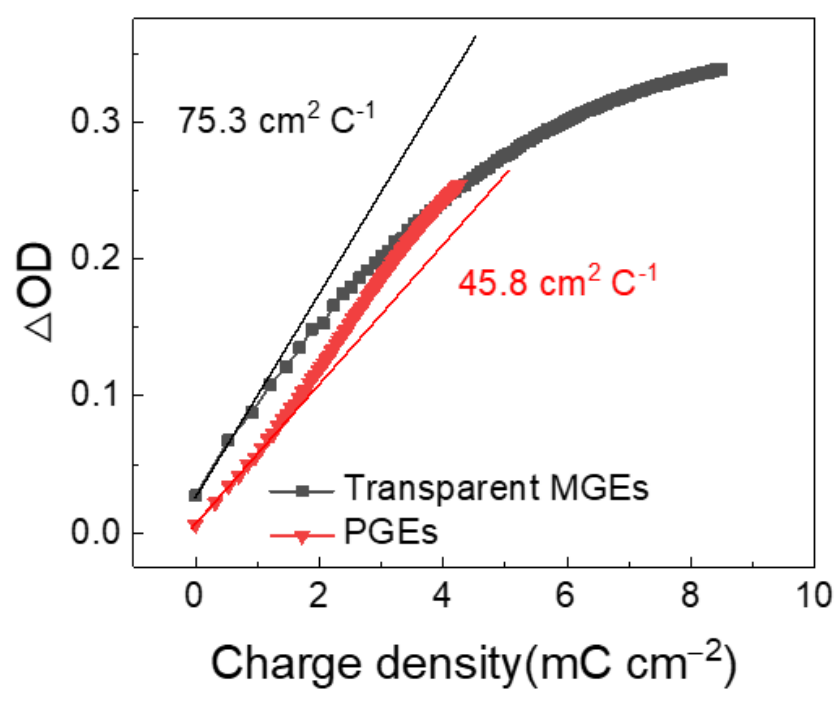

Figure S16. (a) Optical density changes ( $\triangle \mathrm{OD}$ ) as a function of charge densities for two types of EC devices based on MGEs and PGEs, respectively.

Here, $\triangle \mathrm{OD}$ is calculated by the equation of $\Delta \mathrm{OD}=\log \left(T_{b} / T_{c}\right)$, where $T_{b}$ and $T_{c}$ are the transmittances of EC devices under the bleached and colored states, respectively. 
a
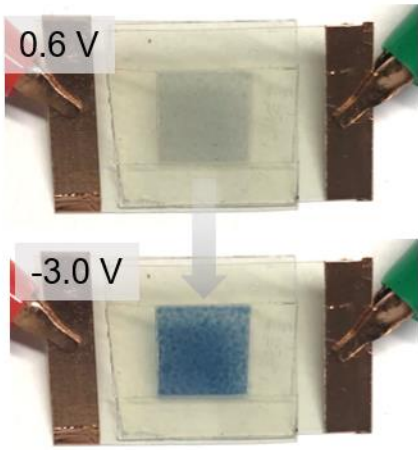

b
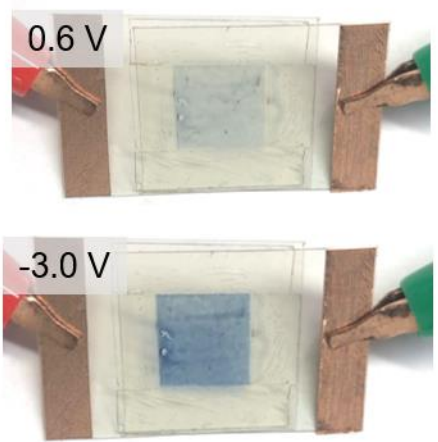

Figure S17. Digital photographs of PEDOT-based EC devices assembled by (a) transparent MGEs and (b) PEGs under different voltages.

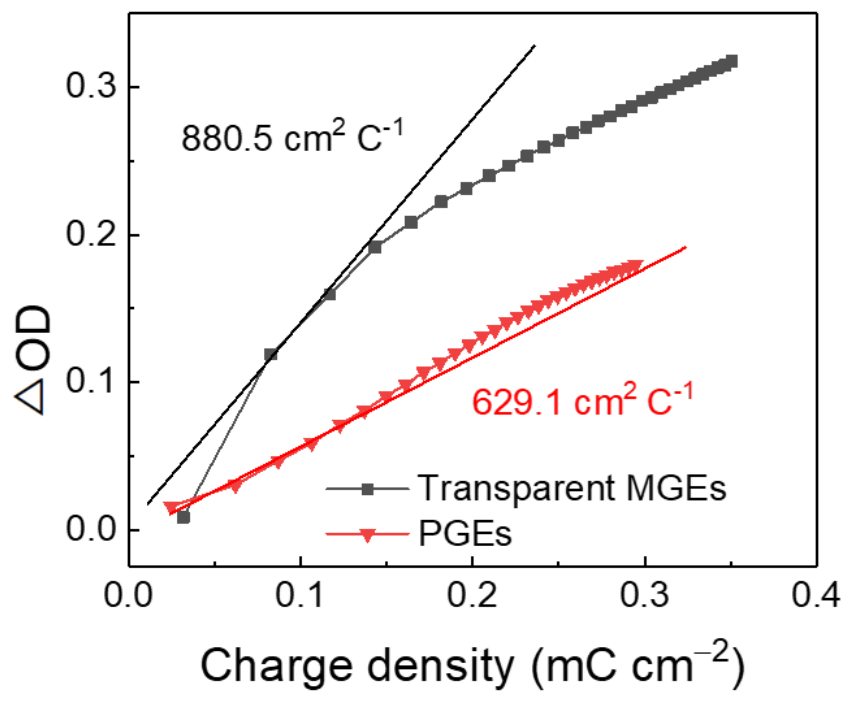

Figure S18. $\triangle O D$ as a function of charge densities for two flexible EC devices. 


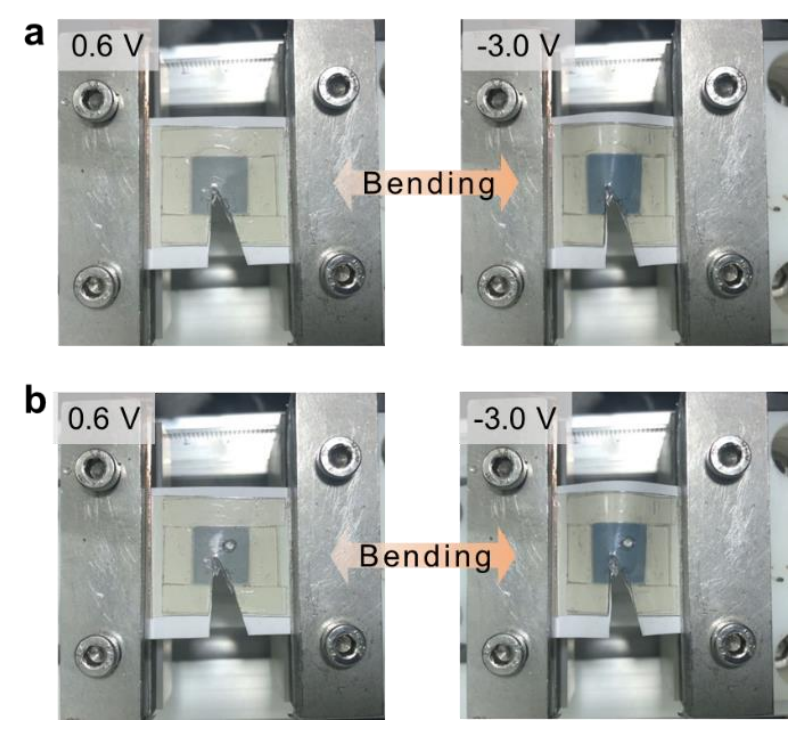

Figure S19. Digital photographs of the EC devices at bleached (left) and colored (right) states: (a) cut EC device; (b) cut and punched device. 
Table S1. Summary of different MOF-based electrolytes under gel or quasi-solid states at the room temperature.

\begin{tabular}{|c|c|c|c|c|c|c|}
\hline Materials & $\begin{array}{c}\text { Transmittance } \\
\text { (\%) }\end{array}$ & $\begin{array}{l}\text { Conductivity } \\
\qquad\left(\mathrm{S} \mathrm{cm}^{-1}\right)\end{array}$ & $\begin{array}{c}\text { Activation } \\
\text { energy }\end{array}$ & $\begin{array}{c}\text { Bending } \\
\text { durability }\end{array}$ & $\begin{array}{l}\text { Applicati } \\
\text { on field }\end{array}$ & $\begin{array}{c}\text { Referen } \\
\text { ce }\end{array}$ \\
\hline $\begin{array}{c}\text { MOF-5 } \\
\text { /LiTFSI/[AMIm]TFSI }\end{array}$ & N.A. & $1.58 \times 10^{-3}$ & $\begin{array}{l}\leq 6 \mathrm{~kJ} \\
\mathrm{~mol}^{-1}\end{array}$ & l & $\begin{array}{c}\text { Non-EC } \\
\text { filed }\end{array}$ & 1 \\
\hline $\begin{array}{c}\mathrm{Al}-\mathrm{BTC} / \mathrm{I}_{2} / \mathrm{LiI} \cdot \\
\mathrm{GuSCN} / \mathrm{TBP} / \mathrm{PMII} / \mathrm{ACN} / \\
\text { Valeronitrile }\end{array}$ & Opaque & $5.8 \times 10^{-3}$ & / & / & $\begin{array}{c}\text { Non-EC } \\
\text { filed }\end{array}$ & 2 \\
\hline $\begin{array}{c}\mathrm{Mg}_{2}(\text { dobpdc }) / \mathrm{Mg}(\mathrm{OPhCF} \\
3)_{2} / \\
\cdot \mathrm{Mg}(\mathrm{TFSI})_{2} / \text { Triglyme }\end{array}$ & Opaque & $2.5 \times 10^{-4}$ & $0.11 \mathrm{eV}$ & / & $\begin{array}{l}\text { Non-EC } \\
\text { filed }\end{array}$ & 3 \\
\hline $\begin{array}{c}\text { MOF-525 } \\
\text { /LiTFSI/[EMIM]TFSI }\end{array}$ & Opaque & $3.0 \times 10^{-4}$ & / & l & $\begin{array}{l}\text { Non-EC } \\
\text { filed }\end{array}$ & 4 \\
\hline $\begin{array}{c}\mathrm{Mg}_{2}(\mathrm{dobdc}) / \mathrm{LiO}^{i} \mathrm{Pr} / \cdot \\
\mathrm{LiBF}_{4} / \mathrm{EC} / \mathrm{DEC}\end{array}$ & Opaque & $3.1 \times 10^{-4}$ & $0.15 \mathrm{eV}$ & l & $\begin{array}{l}\text { Non-EC } \\
\text { filed }\end{array}$ & 5 \\
\hline $\mathrm{MIT}-20 / \mathrm{LiBF}_{4} / \mathrm{PC}$ & Opaque & $4.8 \times 10^{-4}$ & $0.16 \mathrm{eV}$ & / & $\begin{array}{l}\text { Non-EC } \\
\text { filed }\end{array}$ & 6 \\
\hline MOF-688/LiTFSI/PC & Opaque & $3.4 \times 10^{-4}$ & l & l & $\begin{array}{c}\text { Non-EC } \\
\text { filed }\end{array}$ & 7 \\
\hline $\mathrm{MIL}-100-\mathrm{Al} / \mathrm{LiClO}_{4} / \mathrm{PC}$ & Opaque & $1.22 \times 10^{-3}$ & $0.21 \mathrm{eV}$ & l & $\begin{array}{l}\text { Non-EC } \\
\text { filed }\end{array}$ & 8 \\
\hline $\mathrm{MOF}-5 / \mathrm{LiClO}_{4} / \mathrm{PC}$ & Opaque & $1.3 \times 10^{-4}$ & $0.40 \mathrm{eV}$ & l & $\begin{array}{l}\text { Non-EC } \\
\text { filed }\end{array}$ & 8 \\
\hline $\mathrm{H}-\mathrm{MOF} / \mathrm{LiClO}_{4} / \mathrm{PC}$ & 82.2 & $2.66 \times 10^{-3}$ & $0.16 \mathrm{eV}$ & $\begin{array}{l}\text { ca. } 88 \% \text { in } \\
1000 \\
\text { cycles }\end{array}$ & EC filed & $\begin{array}{l}\text { This } \\
\text { work }\end{array}$ \\
\hline
\end{tabular}

Note: MOF-5, Al-BTC, $\mathrm{Mg}_{2}$ (dobpdc), MOF-525, $\mathrm{Mg}_{2}$ (dobdc), MIT-20, MOF-688, and MIL-100-Al are the abbreviations of different MOF materials. 
Table S2. Summary of typical transparent gel electrolytes for EC devices.

\begin{tabular}{|c|c|c|c|c|c|}
\hline Electrolyte & $\begin{array}{l}\text { Conductivity (S } \\
\qquad \mathbf{c m}^{-1} \text { ) }\end{array}$ & $\begin{array}{c}\text { EC } \\
\text { layer }\end{array}$ & $\begin{array}{c}\text { Bending } \\
\text { durability } \\
\text { (1000 cycles) }\end{array}$ & $\begin{array}{c}\text { Optical } \\
\text { contrast }(\triangle \\
T)\end{array}$ & Reference \\
\hline $\mathrm{PMMA} / \mathrm{LiClO}_{4} / \mathrm{PC}$ & $1.7 \times 10^{-3}$ & $\mathrm{WO}_{3}$ & / & $\begin{array}{c}61 \% \text { at } \\
632.8 \mathrm{~nm}\end{array}$ & 9 \\
\hline $\mathrm{PMMA} / \mathrm{SN} / \mathrm{LiClO}_{4} / \mathrm{PC}$ & $1.46 \times 10^{-3}$ & $\mathrm{WO}_{3}$ & / & $\begin{array}{l}52.4 \% \text { at } \\
695 \mathrm{~nm}\end{array}$ & 10 \\
\hline d-PCL(530)/Siloxane/[Emim] $\mathrm{BF}_{4}$ & $4.0 \times 10^{-4}$ & $\mathrm{WO}_{3}$ & l & $\begin{array}{l}8.8 \% \text { at } \\
555 \mathrm{~nm}\end{array}$ & 11 \\
\hline $\mathrm{PVDF}-\mathrm{HFP} / \mathrm{LiCF}_{3} \mathrm{SO}_{3} / \mathrm{ZrO}_{2} / \mathrm{PC}$ & $1.78 \times 10^{-3}$ & PANI:DBSA & l & $\begin{array}{l}\text { ca. } 24 \% \text { at } \\
700 \mathrm{~nm}\end{array}$ & 12 \\
\hline $\mathrm{HPC} / \mathrm{Bu}_{4} \mathrm{NBF}_{4} / \mathrm{DBSA} / \mathrm{PEG}$ & $1.13 \times 10^{-5}$ & PEDOT & l & $\begin{array}{l}35 \% \text { at } \\
650 \mathrm{~nm}\end{array}$ & 13 \\
\hline $\begin{array}{c}\text { PVDF- } \\
\mathrm{HFP} / \mathrm{dmFc} / \mathrm{LiTFSI} /[\text { Emim]TFSI/ }\end{array}$ & / & $\mathrm{WO}_{3}$ & $\begin{array}{l}\text { ca. } 90 \% \\
\text { retention }\end{array}$ & $\begin{array}{l}76 \% \text { at } \\
700 \mathrm{~nm}\end{array}$ & 14 \\
\hline $\mathrm{H}-\mathrm{MOF} / \mathrm{LiClO}_{4} / \mathrm{PC}$ & $2.66 \times 10^{-3}$ & $\mathrm{WO}_{3}$ & l & $\begin{array}{l}30.8 \% \text { at } \\
633 \mathrm{~nm}\end{array}$ & $\begin{array}{l}\text { This } \\
\text { work }\end{array}$ \\
\hline $\mathrm{H}-\mathrm{MOF} / \mathrm{LiClO}_{4} / \mathrm{PC}$ & $2.66 \times 10^{-3}$ & PEDOT & $\begin{array}{l}\text { ca. } 88 \% \\
\text { retention }\end{array}$ & $\begin{array}{l}29.7 \% \text { at } \\
700 \mathrm{~nm}\end{array}$ & $\begin{array}{l}\text { This } \\
\text { work }\end{array}$ \\
\hline
\end{tabular}




\section{Supporting References}

(1) Singh, A.; Vedarajan, R.; Matsumi, N. Modified Metal Organic Frameworks (MOFs)/Ionic Liquid Matrices for Efficient Charge Storage. J. Electrochem. Soc. 2017, 164 (8), H5169-H5174.

(2) Fan, J.; Li, L.; Rao, H.-S.; Yang, Q.-L.; Zhang, J.; Chen, H.-Y.; Chen, L.; Kuang, D.-B.; Su, C.-Y. A Novel Metal-Organic Gel Based Electrolyte for Efficient Quasi-Solid-State Dye-Sensitized Solar Cells. J. Mater. Chem. A 2014, 2 (37), 15406-15413.

(3) Aubrey, M. L.; Ameloot, R.; Wiers, B. M.; Long, J. R. Metal-Organic Frameworks as Solid Magnesium Electrolytes. Energ. Environ. Sci. 2014, 7 (2), 667-671.

(4) Wang, Z.; Tan, R.; Wang, H.; Yang, L.; Hu, J.; Chen, H.; Pan, F. A Metal-Organic-Framework-Based Electrolyte with Nanowetted Interfaces for High-Energy-Density Solid-State Lithium Battery. Adv. Mater. 2018, 30 (2), 1704436.

(5) Wiers, B. M.; Foo, M.-L.; Balsara, N. P.; Long, J. R. A Solid Lithium Electrolyte via Addition of Lithium Isopropoxide to A Metal-Organic Framework with Open Metal Sites. J. Am. Chem. Soc. 2011, 133 (37), 14522-14525.

(6) Park, S. S.; Tulchinsky, Y.; Dincă, M. Single-Ion $\mathrm{Li}^{+}, \mathrm{Na}^{+}$, and $\mathrm{Mg}^{2+}$ Solid Electrolytes Supported by A Mesoporous Anionic Cu-Azolate Metal-Organic Framework. J. Am. Chem. Soc. 2017, 139 (38), 13260-13263.

(7) Xu, W.; Pei, X.; Diercks, C. S.; Lyu, H.; Ji, Z.; Yaghi, O. M. A Metal-Organic Framework of Organic Vertices and Polyoxometalate Linkers as A Solid-State Electrolyte. J. Am. Chem. Soc. 2019, 141 (44), 17522-17526.

(8) Shen, L.; Wu, H. B.; Liu, F.; Brosmer, J. L.; Shen, G.; Wang, X.; Zink, J. I.; Xiao, Q.; Cai, M.; Wang,

G. Creating Lithium-Ion Electrolytes with Biomimetic Ionic Channels in Metal-Organic Frameworks. Adv. Mater. 2018, 30 (23), 1707476.

(9) Tang, Q.; Li, H.; Yue, Y.; Zhang, Q.; Wang, H.; Li, Y.; Chen, P. 1-Ethyl-3-Methylimidazolium Tetrafluoroborate-Doped High Ionic Conductivity Gel Electrolytes with Reduced Anodic Reaction Potentials for Electrochromic Devices. Mater. Design 2017, 118, 279-285.

(10) Wang, J.-Y.; Wang, M.-C.; Jan, D.-J. Synthesis of Poly(Methyl Methacrylate)-Succinonitrile Composite Polymer Electrolyte and Its Application for Flexible Electrochromic Devices. Sol. Energ. Mat. Sol. Cells 2017, 160, 476-483.

(11) Fernandes, M.; Leones, R.; Costa, A. M. S.; Silva, M. M.; Pereira, S.; Mano, J. F.; Fortunato, E.; Rego, R.; de Zea Bermudez, V. Electrochromic Devices Incorporating Biohybrid Electrolytes Doped with A Lithium Salt, An Ionic Liquid or A Mixture of Both. Electrochim. Acta. 2015, 161, 226-235.

(12) Puguan, J. M. C.; Chung, W.-J.; Kim, H. Ion-Conductive and Transparent PVdF-HFP/SilaneFunctionalized $\mathrm{ZrO}_{2}$ Nanocomposite Electrolyte for Electrochromic Applications. Electrochim. Acta. 2016, 196, 236-244.

(13) Ledwon, P.; Andrade, J. R.; Lapkowski, M.; Pawlicka, A. Hydroxypropyl Cellulose-Based Gel Electrolyte for Electrochromic Devices. Electrochim. Acta. 2015, 159, 227-233.

(14) Yun, T. Y.; Li, X.; Bae, J.; Kim, S. H.; Moon, H. C. Non-Volatile, Li-Doped Ion Gel Electrolytes for Flexible $\mathrm{WO}_{3}$-Based Electrochromic Devices. Mater. Design 2019, 162, 45-51. 